\title{
Severe Hypertriglyceridemia Secondary to Uncontrolled Diabetes Mellitus Successfully Managed with Insulin
}

\author{
Balasingam Nisahan ${ }^{1}$, Nay Lwin Htet ${ }^{1}$, Joseph Pratheepan ${ }^{1}$ \\ ${ }^{1}$ Department of Medicine Hervey Bay Hospital Queensland Australia
}

\begin{abstract}
Hypertriglyceridemia is one of the common lipid abnormalities in patients with type 2 diabetes mellitus. It is extremely rare to develop severe hypertriglyceridemia in patients with diabetes mellitus on fibrates without any other risk factors for hypertriglyceridemia. Acute pancreatitis is the main consequence of severe hypertriglyceridemia. We report a case of severe hypertriglyceridemia in a patient with uncontrolled type 2 diabetes, on fibrate and was successfully treated with intravenous insulin infusion.
\end{abstract}

\section{Key words}

Severe hypertriglyceridemia, Lipoprotein lipase, Insulin, Diabetes Mellitus

\section{Introduction}

Diabetes mellitus is a metabolic disorder in which, deficiency of insulin causes increased release of free fatty acids from adipose tissue and amino acids from muscles while activated counterregulatory hormones lead to glycogenolysis and gluconeogenesis in the liver. Liver utilises the excess free fatty acid which leads to increased production of VLDL. This in turn causes hypertriglyceridemia (1). The main concern of severe hypertriglyceridemia is acute pancreatitis. Hypertriglyceridemia accounts for $1-7 \%$ of all cases of pancreatitis (2). There is no standard treatment guideline for severe hypertriglyceridemia. Intravenous insulin treatment may be an effective first line treatment for severe hypertriglyceridemia.

\section{Case report}

A 42year-old female presented with multiple joint pains, abdominal pain and loose stools for one-day duration. She also had bifrontal headache with photophobia without any signs of meningism.
Her visual acuity and visual field were normal. She had a past medical history of type 2diabetes mellitus, dyslipidaemia, gastro-oesophageal reflux disease, depression and bronchial asthma. Her long-term oral medications were metformin $500 \mathrm{mg}$ mane and fenofibrate $145 \mathrm{mg}$ mane. She was not consuming alcohol in excess. She was very keen to avoid fatty meals since she was diagnosed with dyslipidaemia. She did not have a family history of hypertriglyceridemia in her first or second-degree relatives. Her BMI was $31.25 \mathrm{~kg} / \mathrm{m}^{2}$. She did not have any clinical features of hyperlipidaemia such as eruptive xanthomas or lipemia retinalis. Her haemodynamic status was normal. Her abdominal examination revealed mild epigastric tenderness without guarding or rigidity.

Her blood investigations revealed a lipemic sample (Fig:1) with triglycerides of $117.4 \mathrm{mmol} / \mathrm{L}$ (Normal value $<1.5 \mathrm{mmol} / \mathrm{L})$. Other lipid parameters were not analysed because of the lipemic serum. (Figure : 1)

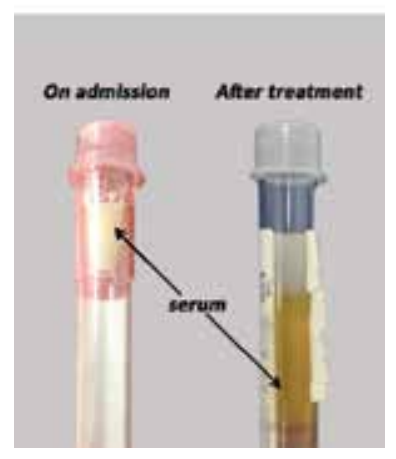

Fig:1 Appearance of serum before and after insulin infusion.

Her renal function and electrolytes were normal. Serum amylase and lipase were normal. Her liver function revealed ALT of $35 \mathrm{U} / \mathrm{L}, \mathrm{AST}$ of $31 \mathrm{U} / \mathrm{L}$ and gamma GT was 56U/L. Alkaline phosphatase and serum proteins and coagulations studies were normal. Her thyroid function was normal and her 
HbA1c was 9.6\%. Blood glucose on admission was $20.3 \mathrm{mmol} / \mathrm{L}$. Vasculitis screening; Rheumatoid factor and anti-CCP were negative. Computed tomography of the abdomen showed enlarged fatty liver. She was managed with insulin infusion for 2 weeks as per protocol with intravenous dextrose to avoid hypoglycaemia. Her fenofibrate was continued with 3 grams of fish oil. She was discharged on fibrates and niacin. Follow up at one month showed the triglycerides of $3.4 \mathrm{mmol} / \mathrm{L}$.

\section{Discussion}

Primary hypertriglyceridaemia may be familial. Secondary hypertriglyceridaemia is mainly due to uncontrolled diabetes mellitus, obesity, excess alcohol consumption or oestrogen therapy. More complex forms of hypertriglyceridaemia develop with hypothyroidism, end stage renal failure, nephrotic syndrome, HIV infection and antiretroviral therapies. Severe hypertriglyceridemia is defined as triglycerides above $22.4 \mathrm{mmol} / \mathrm{L}$ $(2000 \mathrm{mg} / \mathrm{dL})$ and it is almost always having both secondary and genetic forms of hypertriglyceridemia (3). Severe hypertriglyceridaemia of more than $11.2 \mathrm{mmol} / \mathrm{L}(1000 \mathrm{mg} / \mathrm{dL})$ needs urgent treatment to reduce the risk of pancreatitis (4). Possible elimination of secondary causes and control of diabetes are important in preventing hypertriglyceridemia. Avoidance of simple sugar is important in controlling postprandial hypertriglyceridemia. Few case reports mentioned that rapid reduction of severe hypertriglyceridemia was achieved with heparin and insulin infusion. Plasma paresis with salt free human albumin or fresh frozen plasma is also an effective method in correcting hypertriglyceridemia rapidly (5). For long term treatment, fibrates, Omega-3 fatty acids supplement and low-fat diet are effective.

Removal of triglycerides is mediated by lipoprotein lipase enzyme which is produced in adipocytes and myocytes. Lipoprotein lipase hydrolyse triglycerides in to glycerol and free fatty acids. Also, it facilitates free fatty acid storage in adipocytes (6). Heparin promotes translocation of lipoprotein lipase from tissues to capillary endothelium because lipoprotein lipase is a high affinity heparin binding protein. Carboxy terminal binding of heparin is needed to maintain lipoprotein lipase activity and to prevent inactivation.

This patient had diabetes and obesity as risk factors without any family history and she was already taking fibrates. In conclusion, insulin infusion is an effective, non-invasive and safe method to treat severe hypertriglyceridemia in addition to the oral pharmacological treatment. Early identification and rapid reduction of high serum triglycerides level are extremely important to avoid life-threatening complications like acute pancreatitis.

\section{References}

1. Hahn, S.J., Park, J.H., Lee, J.H., Lee, J.K. and Kim, K.A., 2010. Severe hypertriglyceridemia in diabetic ketoacidosis accompanied by acute pancreatitis: case report. Journal of Korean medical science, 25(9), pp.1375-1378.

2. Yadav, D. and Pitchumoni, C.S., 2003. Issues in hyperlipidemic pancreatitis. Journal of clinical gastroenterology, 36(1), pp.54-62.

3. Yuan, G., Al-Shali, K.Z. and Hegele, R.A., 2007. Hypertriglyceridemia: its etiology, effects and treatment. Cmaj, 176(8), pp.1113-1120.

4. Brunzell, J.D. and Schrott, H.G., 1973. The interaction of familial and secondary causes of hypertriglyceridemia: role in pancreatitis. Transactions of the Association of American Physicians, 86, pp.245-254.

5. Lams, E., 2006. Pancreatitis and hypertriglyceridaemia. European journal of anaesthesiology, 23(12), pp.1067-1068.

6. Poonuru, S., Pathak, S.R., Vats, H.S. and Pathak, R.D., 2011. Rapid reduction of severely elevated serum triglycerides with insulin infusion, gemfibrozil and niacin. Clinical medicine \& research, 9(1), pp.3841. 